\title{
Fecal lactoferrin in discriminating inflammatory bowel disease from Irritable bowel syndrome: a diagnostic meta-analysis
}

\author{
Xing-lu Zhou', Wen Xu' ${ }^{1}$ Xiao-xiao Tang ${ }^{1}$, Lai-sheng Luo ${ }^{1}$, Jiang-feng Tu', Chen-jing Zhang ${ }^{1}$, Xiang Xu' \\ Qin-dong Wu ${ }^{1}$ and Wen-sheng Pan ${ }^{1 *}$
}

\begin{abstract}
Background: To perform a meta-analysis evaluating the diagnostic ability of fecal lactoferrin ( $F L$ ) to distinguish inflammatory bowel disease (IBD) from irritable bowel syndrome (IBS).

Methods: The Medline, EMBASE, Web of Science, Cochrane library and CNKI databases were systematically searched for studies that used FL concentrations to distinguish between IBD and IBS. The sensitivity, specificity, and other diagnostic indexes of FL were pooled using a random-effects model.

Results: Seven studies, involving 1012 patients, were eligible for inclusion. In distinguishing IBD from IBS, FL had a pooled sensitivity of 0.78 ( $95 \%$ confidence interval [CI]: $0.75,0.82$ ), a specificity of 0.94 (95\% Cl: 0.91, 0.96), a positive likelihood ratio of 12.31 (95\% Cl: 5.93, 29.15), and a negative likelihood ratio of 0.23 (95\% Cl: $0.18,0.29)$. The area under the summary receiver-operating characteristic curve was $0.94(95 \% \mathrm{Cl}: 0.90,0.98)$ and the diagnostic odds ratio was 52.65 (95\% Cl: 25.69, 107.91).
\end{abstract}

Conclusions: $\mathrm{FL}$, as a noninvasive and simple marker, is useful in differentiating between IBD and IBS.

Keywords: Fecal lactoferrin, Inflammatory bowel disease, Irritable bowel syndrome, Meta-analysis

\section{Background}

Inflammatory bowel disease (IBD) and irritable bowel syndrome (IBS) are common conditions that may present with a similar symptom complex of abdominal pain and altered bowel habits. However, the two conditions differ markedly in their pathophysiology, prognosis and therapeutic approaches. IBD represents a group of idiopathic, chronic, inflammatory intestinal conditions [1], commonly requires a lifetime of medical care, and can even cause significant morbidity. In contrast, IBS is a chronic, functional gastrointestinal disorder without inflammation [2], with most IBS patients having a favorable prognosis. Despite existing diagnostic criteria, such as the Rome criteria, it remains difficult to distinguish IBD from IBS using symptoms and signs only, especially in the absence of rectal bleeding and systemic illness. As clinical differentiation

\footnotetext{
*Correspondence: wspan223@163.com

'Department of Gastroenterology, Second Affiliated Hospital of Zhejiang

University, School of Medicine, 88 Jiefang Road, Hangzhou, China

Full list of author information is available at the end of the article
}

remains challenging and may delay effective treatment, most patients with IBS are evaluated by endoscopy and radiographic imaging to exclude a diagnosis of IBD. This not only exposes patients to the inherent risks associated with these procedures, but increases their economic burden [3]. Less invasive and less expensive diagnostic methods are needed to more effectively rule out IBD in primary care patients with chronic abdominal complaints.

Fecal measurements of potential biomarkers are noninvasive diagnostic tests for intestinal mucosal inflammation and may correlate well with disease activity. Several neutrophil-granular proteins released by activated neutrophils may constitute fecal markers of intestinal inflammation, including lactoferrin (LF), calprotectin (Cal), polymorphonuclear neutrophil-elastase (PMN-e), and lysozyme (Lys) [4], with Cal and LF appearing to be the most promising surrogate biomarkers. $\mathrm{Cal}$ is a calcium- and zinc-binding protein that constitutes up to $60 \%$ of the total cytosolic protein content of neutrophils [5]. Cal has been shown to reflect neutrophil migration in the intestines of 
IBD patients and measurements of this protein may be an alternative to ${ }^{111}$ indium labeled radioactive techniques [6]. LF is an iron binding glycoprotein with a molecular mass of about $80 \mathrm{kDa}$ that is present in various secretory fluids, such as milk, saliva, tears, and nasal secretions [7]. LF is a component of the innate immune system, with antimicrobial activity as a bactericide and fungicide, as well as being a major constituent of neutrophil granules that is released during apoptosis [8]. During intestinal inflammation polymorphonuclear neutrophils infiltrate the mucosa, increasing LF concentration in feces proportional to neutrophil translocation to the GI tract [9]. Studies investigating whether FL can be used as a noninvasive marker to distinguish IBD from non inflammatory conditions, especially IBS, have yielded variable results [3,10-18]. We therefore designed a meta-analysis to assess the overall diagnostic capacity of FL in discriminating IBD from IBS.

\section{Methods}

\section{Literature search}

Medline (using PubMed as the search engine), EMBASE, Web of science, Cochrane library and China National Knowledge Infrastructure (CNKI) databases were searched by two reviewers (XZ and WX) independently for relevant articles published in English and Chinese up to November 2013. The MeSH headings and key words used were "fecal lactoferrin", "lactoferrin and inflammatory bowel disease", "lactoferrin and irritable bowel syndrome", "IBD and IBS", and "lactoferrin and intestinal inflammation". Further searches included combinations of "lactoferrin" with "enteritis", "ileitis", "enteritidis", and "esoenteritis". We also screened the reference lists of included studies and review articles. The results were then hand searched for eligible studies.

\section{Study eligibility}

Studies were included if they met the following criteria: 1) assessed the diagnostic performance of FL in discriminating IBD from IBS; 2) used endoscopic and histological methods as the reference standards; 3) presented sufficient information to calculate the true-positive (TP), false-positive (FP), true-negative (TN), and false-negative (FN) rates; and 4) were conducted on human subjects including pediatric or adult population. Letters, reviews, conference abstracts, comments and case reports were excluded because of the limited data presented. When the same population was reported in two or more publications, only the most informative or complete study was included to avoid duplication of information.

\section{Data extraction and quality assessment}

The same reviewers (XZ and WX) independently retrieved specific data from each full-text article using a standard data extraction form, which included author, year of publication, nation, patient characteristics, FL assay, and cutoff value. The published values for TP, FP, TN, and FN were extracted and used to construct a $2 \times$ 2 contingency table. Disagreement between the two reviewers was resolved by consensus. If consensus among the two reviewers could not be reached, a third investigator (XT) was referred to for arbitration and consensus. The methodological quality of the included studies was evaluated using the Quality Assessment of Diagnostic Accuracy Studies (QUADAS) list [19], which consists of 14 items scored as "yes", "no" or "unclear".

\section{Statistical analysis}

Statistical analyses were performed using Meta-Disc software version 1.4 (for Windows; XI Cochrane Colloquium; Barcelona, Spain) and STATA statistical software version 12.0 (StataCorp; College Station, TX, USA). For each study, the following indexes of test accuracy were computed by constructing a $2 \times 2$ contingency table: sensitivity, specificity, positive likelihood ratio (PLR), negative likelihood ratio (NLR), and diagnostic odds ratio (DOR). A random-effects model was used to calculate the pooled diagnostic indices, with corresponding 95\% confidence intervals (CIs), across studies. A summary receiver operating characteristic (sROC) curve was plotted to determine the relationship between sensitivity and specificity, and the area under the curve (AUC), an analytical summary of the test performance, was determined, with the upper and lower limits of the 95\% CI of the AUC calculated as AUC + 1.96 Se and AUC-1.96 Se, respectively. Heterogeneity was assessed by determining the Cochran Q-statistic and by the test of inconsistency $\left(I^{2}\right)$ [20], with a $P$-value $<0.05$ or $I^{2}>50 \%$ suggesting significant heterogeneity [21]. A random effects model, which considered both within- and between-study variations [22], was used throughout because of the observational nature of most studies. In addition, the Spearman's rank correlation coefficient was calculated to assess the threshold effect. Finally publication bias was determined using Egger precision weighted linear regression tests [23].

\section{Results}

The initial literature search identified a total of 311 reports; of these, 270 were excluded based on their titles and abstracts (Figure 1). A review of the full text of the remaining 41 articles led to an additional 34 being excluded because of failure to meet the inclusion criteria, duplicate publication or not reporting the data required to create a contingency table. Finally, seven eligible studies were included [3,10-15] (Table 1).

The seven eligible studies included a total of 1336 patients, including 737 with IBD, 381 with IBS, and 218 healthy volunteers, who underwent FL testing. One study 


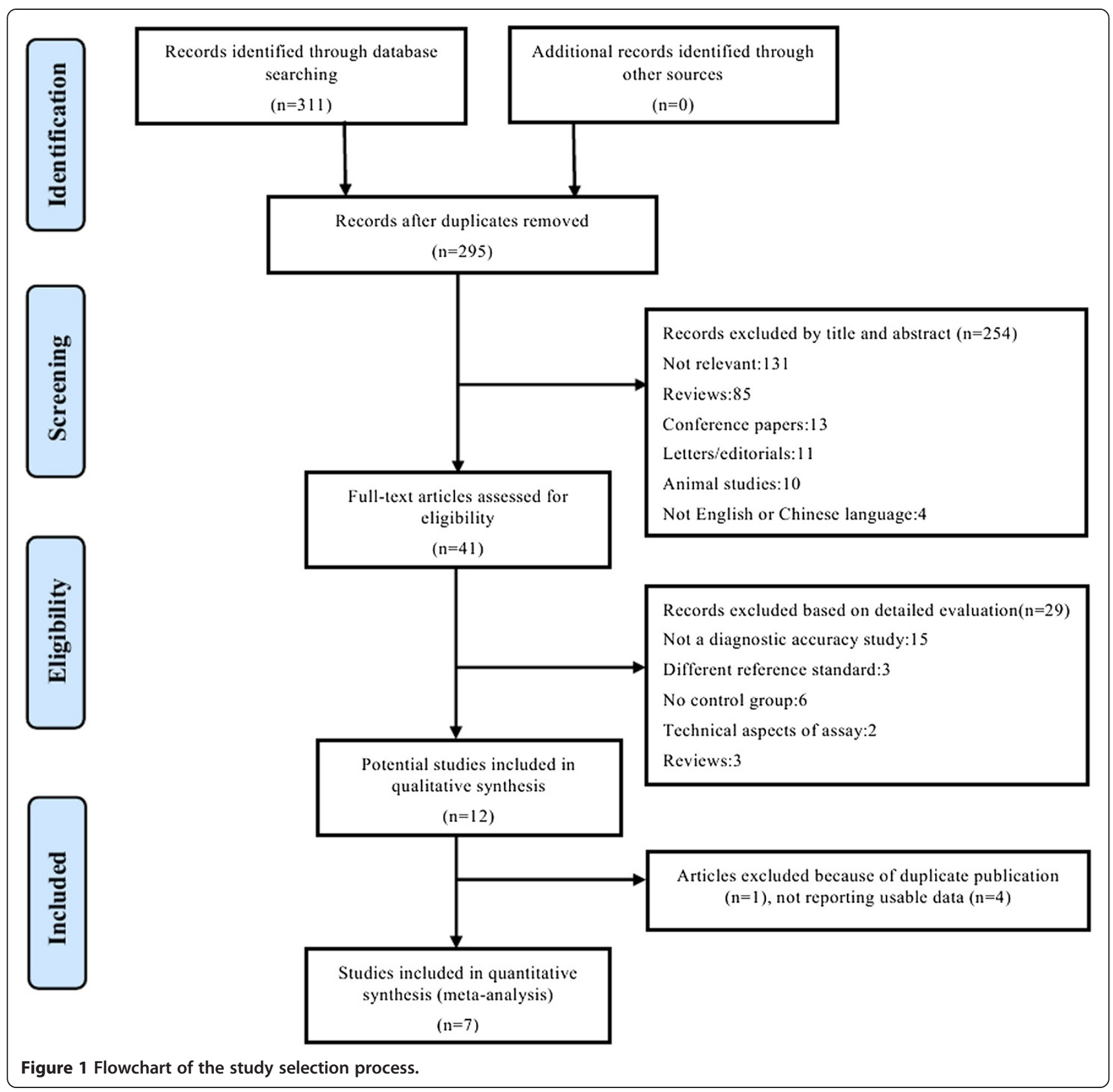

found that mean FL level was similar in patients with IBS and healthy control; that study combined these two groups for comparisons with patients with IBD [10]. Another study only reported the TP, FP, TN, and FN rates of FL assay in distinguishing active IBD from IBS [14]. As our aim was to observe the diagnostic performance of FL in differentiating IBD from IBS, our meta-analysis included 1012 patients, 609 with IBD, 381 with IBS and 22 healthy volunteers. Of the seven included studies, one measured FL solely in children and young adults [10], three measured FL in both children and adults [12-14], and the other three measured FL in adults only $[3,11,15]$. All seven studies used a commercially available enzyme-linked immunosorbent assay (IBD-SCAN ${ }^{\bullet}$, Techlab, Blacksburg, VA, USA) to measure FL. The methodological quality of the included studies is presented in Figure 2. Assessment of each risk of bias item is shown as a percentage across all of the included studies (Figure 3).

Figure 4(A) shows the Forest plot for sensitivity and Figure 4(B) shows the Forest plot for specificity of FL in discriminating between IBD and IBS. Sensitivities ranged from 0.69 to 0.86 (pooled sensitivity 0.78 ; 95\% CI: 0.75 , 0.82 ) and specificities from 0.83 to 1.00 (pooled specificity 0.94 ; $95 \%$ CI: $0.91,0.96)$. The pooled PLR was 12.31 
Table 1 Characteristics of the studies included in the meta-analysis

\begin{tabular}{|c|c|c|c|c|c|c|c|c|c|c|c|c|c|c|c|c|}
\hline \multirow[t]{2}{*}{ Study } & \multirow[t]{2}{*}{ Year } & \multirow{2}{*}{$\begin{array}{l}\text { Age(mean/ } \\
\text { range) }\end{array}$} & \multirow[t]{2}{*}{ Nation } & \multicolumn{5}{|c|}{ Number of patients } & \multirow[t]{2}{*}{ FL assay } & \multirow[t]{2}{*}{ Cut off } & \multicolumn{4}{|c|}{ Test result } & \multirow[t]{2}{*}{ SEN (95\% Cl) } & \multirow[t]{2}{*}{ SPE $(95 \% \mathrm{Cl})$} \\
\hline & & & & IBD & 118 & CD & IBS & control & & & TP & FP & $\mathrm{FN}$ & $\overline{\mathrm{N}}$ & & \\
\hline ainct & 007 & 13.4 & IICT & 141 & 62 & 79 & 7 & 22 & IBD-SCAN & $/ \mathrm{ml}$ & 118 & 1 & 23 & $28^{\ddagger}$ & & 0.97 \\
\hline hoe & 2008 & $20-79$ & d & 64 & 28 & 36 & 30 & 42 & $\mathrm{IBD}$ & $7.0 \mathrm{u}$ & 55 & 1 & 9 & 29 & .93) & 0.97 \\
\hline Langhorst & 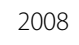 & 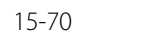 & Germany & 85 & 4 & 43 & 54 & - & v & 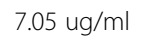 & 68 & 1 & 17 & 45 & 3) & $0.83(0.71-0.9)$ \\
\hline ane & 2003 & $10-78$ & mer & 149 & 71 & 78 & 31 & 56 & IBD-SCAN & $4 \mathrm{ug} / \mathrm{g}$ & 111 & 0 & 38 & 31 & 0.74 & 1.00 \\
\hline . & 2007 & 75 & 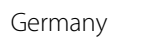 & 45 & 20 & 25 & 31 & - & N & 72 & 37 & 0 & 8 & 87 & 2) & $10 \mathrm{C}$ \\
\hline idhu & 2010 & $42 / 58 / 56^{*}$ & UK & 230 & 126 & 104 & 137 & 98 & IBD-SCAN & $7.25 \mathrm{ug} / \mathrm{g}$ & $70^{\S}$ & 6 & 32 & 131 & $0.69(0.59-0.77)$ & $0.96(0.91-0$. \\
\hline tten & 2008 & $52.3 / 44.5^{\dagger}$ & Netherlands & 23 & - & - & 91 & - & IBD-SCAN & $7.25 \mathrm{mg} / \mathrm{ml}$ & 18 & 9 & 5 & 82 & $0.78(0.56-0.93)$ & $0.90(0.82-0.9$ \\
\hline
\end{tabular}

*Numbers are mean values for IBS, UC and CD respectively. ${ }^{\dagger}$ Numbers are mean values for IBS and IBD respectively.

${ }^{\ddagger}$ Include 22 healthy volunteers. ${ }^{\S}$ Only calculated active IBD.

(95\% CI: 6.05, 25.07; Figure 4(C)), the pooled NLR was 0.23 (95\% CI: 0.18, 0.29; Figure 4(D)), and the pooled DOR was 52.65 (95\% CI: 25.69, 107.91; Figure 5). The chi-square values for sensitivity, specificity, PLR, NLR and DOR were $11.93(P=0.063), 18.68(P=0.005), 15.01$ $(P=0.020), 11.35(P=0.078)$, and $9.49(P=0.148)$, respectively and their $I^{2}$ values were $49.7 \%, 67.9 \%, 60.0 \%$,
$47.1 \%$, and $36.8 \%$, respectively. Significant heterogeneity among were observed for specificity and PLR.

The Spearman's rank correlation coefficient, performed as a test for threshold effect, was found to be -0.198 $(P=0.670)$, indicating that no threshold effect could have caused variations in accuracy estimates among the individual studies.

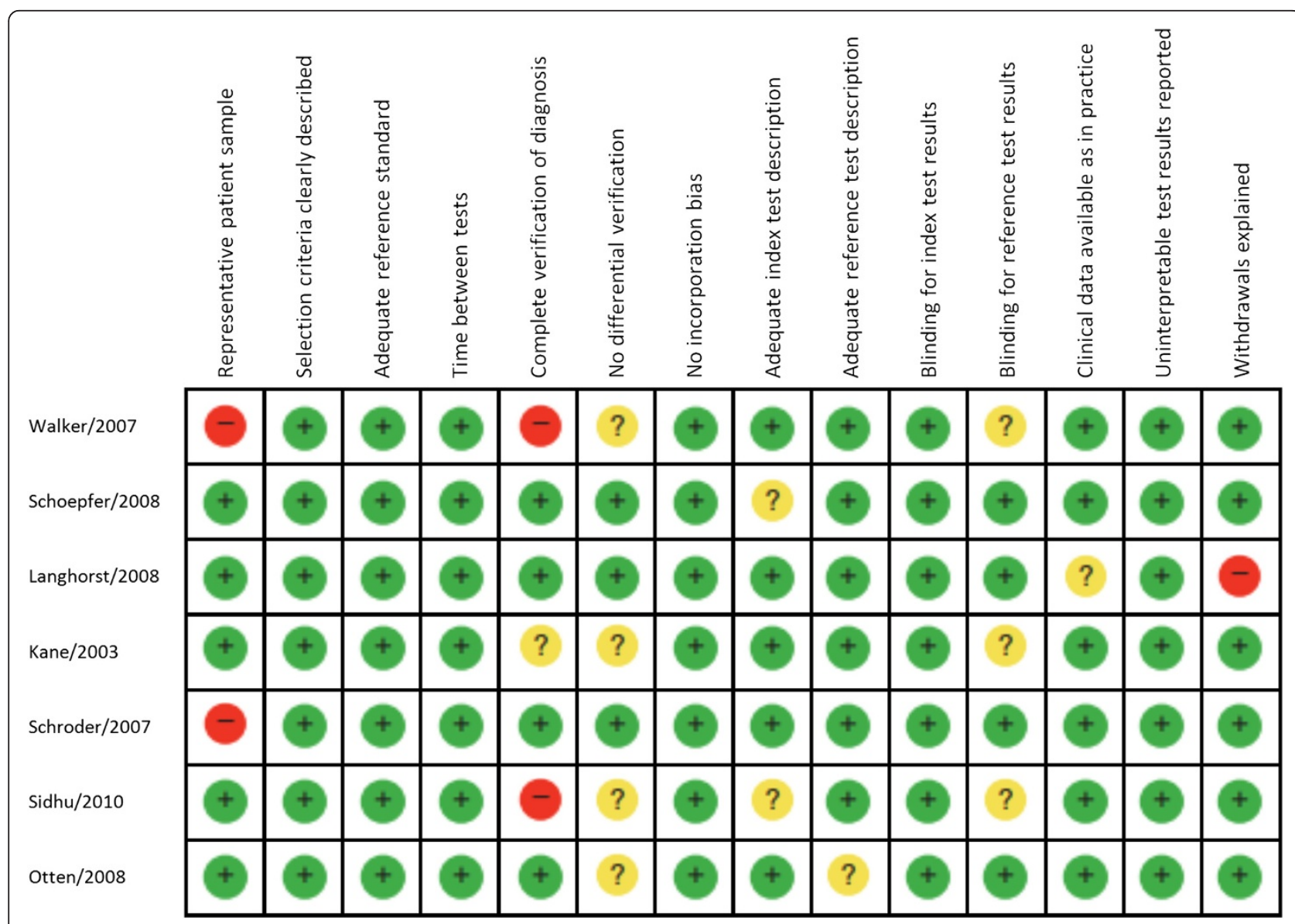

Figure $\mathbf{2}$ Methodological quality of each included study. +, Yes; -, No; ?, unclear. 


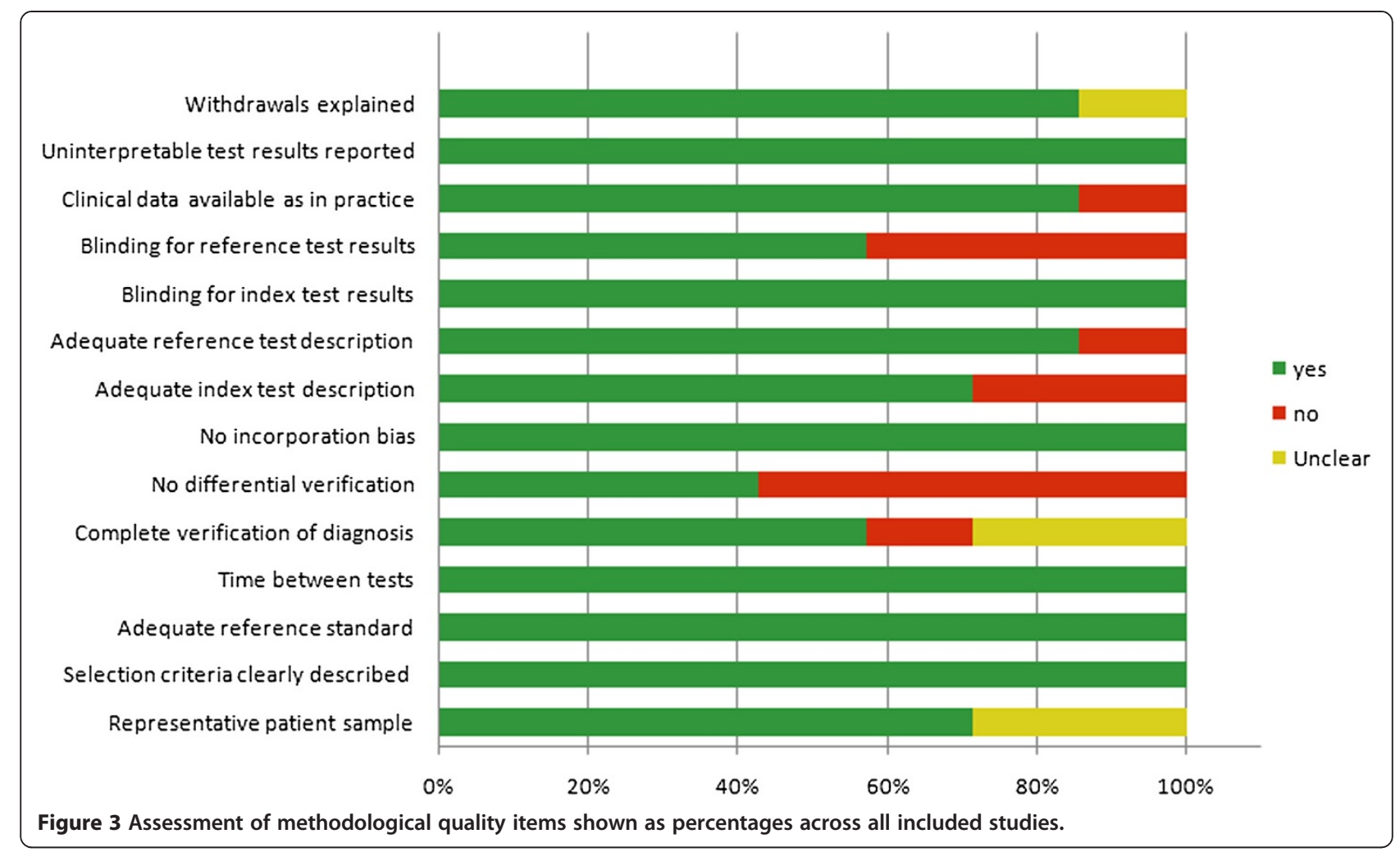

The pooled sROC (Figure 6) showed an AUC of 0.94 with a standard error (SE) of 0.02 . The $95 \%$ CI was 0.90-0.98, while the pooled diagnostic accuracy ( $\left.Q^{*}\right)$ was 0.88 (SE 0.01), demonstrating that FL was highly able to differentiate between IBD and IBS.

The Egger test showed that the potential publication bias was significant $(P<0.05)$. Owing to the limited number of studies included in our meta-analysis, funnel plots were not assessed.

\section{Discussion}

FL assays may detect mucosal inflammatory activity at a level insufficient to cause increases in erythrocyte sendimentation rate (ESR) and C-reactive protein (CRP). FL levels are apparently unaffected by a variety of nonintestinal conditions that elevate markers of systemic inflammation [24]. Lactoferrin is highly stable in feces for up to 7 days at room temperature [9], enabling patients to conveniently collect samples at home and transport

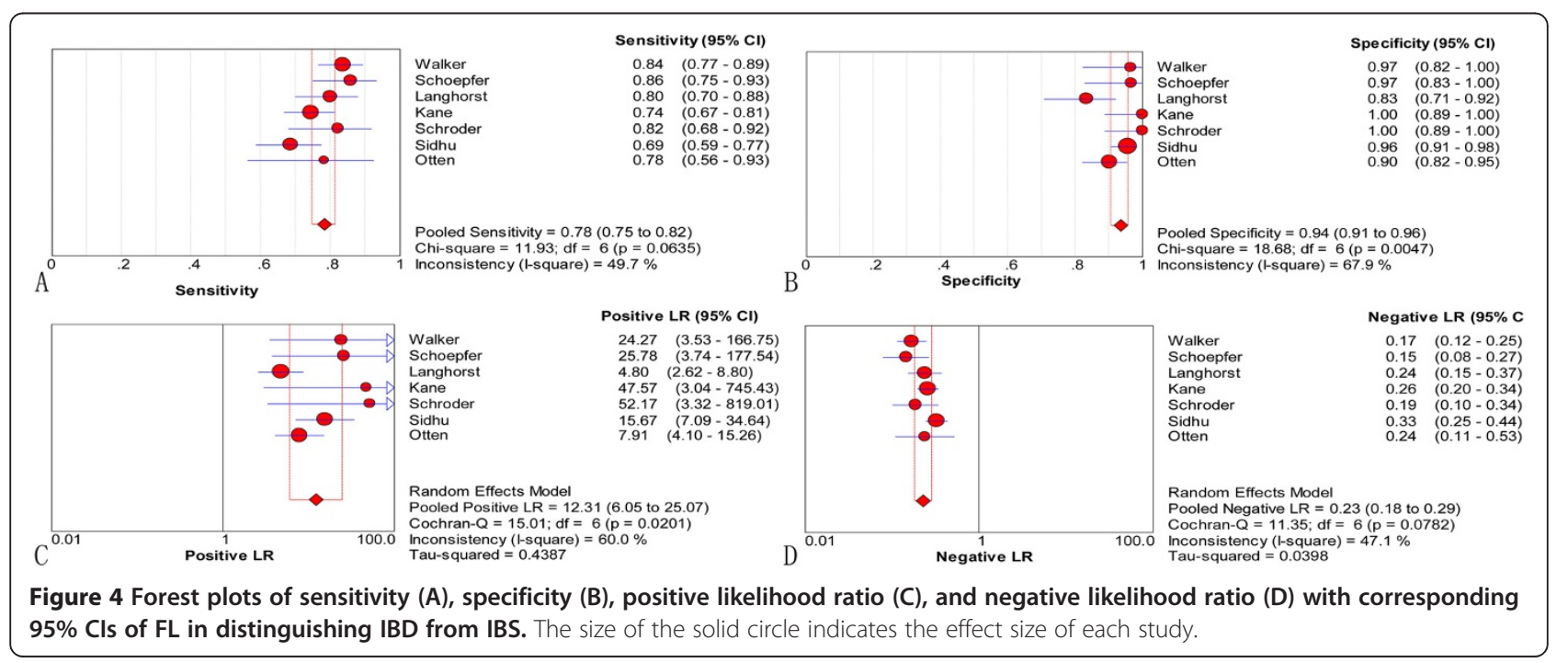




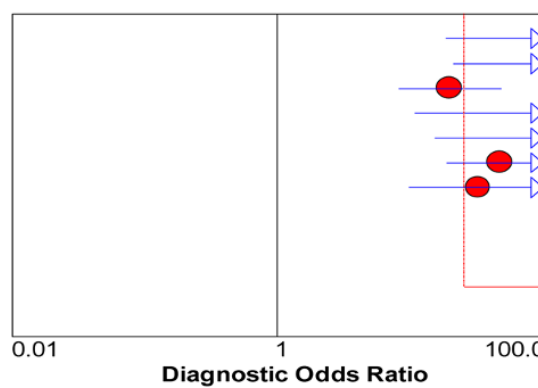

Walker

Schoepfer

Langhorst

Kane

Schrode

Sidhu

Otten

Random Effects Model

Pooled Diagnostic Odds Ratio $=52.65$ (25.69 to 107.91)

Cochran- $Q=9.49 ; \mathrm{df}=6(\mathrm{p}=0.1479)$

Diagnostic Odds Ratio

Figure 5 Forest plots of diagnostic odds ratio with corresponding $95 \%$ Cls of FL in distinguishing IBD from IBS.

them to distant hospitals or laboratories [5]. Moreover, quantitative FL levels can be easily and reliably measured by commercially available enzyme-linked immunoabsorbent assay kits that have been approved by the US Food and Drug Administration [5,25]. FL concentrations have been found to correlate well with endoscopic and histological activity [4,26-29]. Moreover, FL measurements can be used to evaluate responses to anti TNF- $\alpha$ treatment $[30,31]$ or to predict relapse in IBD patients $[10,32]$. Studies have sought to evaluate the accuracy of FL in the diagnosis of organic (versus functional) intestinal diseases, mainly to discriminate IBD from non inflammatory conditions, especially IBS. Few studies to date have directly compared the diagnostic performance of fecal lactoferrin and Cal tests, with some reporting that both are similar in their ability to detect intestinal inflammation $[11,12,29]$, while others found that Cal $[3,17]$ or FL [33] is more accurate diagnostically. LF seems to be more stable at room temperature and therefore may be preferred if long delays are expected before analysis. Age-related variability seems to be less pronounced with lactoferrin and therefore may be preferable in pediatric patients [34]. To our knowledge, this study is the first meta-analysis to assess the diagnostic performance of FL in differentiating between IBD and IBS.

The present meta-analysis demonstrated that FL level had a pooled sensitivity of 0.78 and a pooled specificity of 0.94 in distinguishing IBD (active and inactive) from

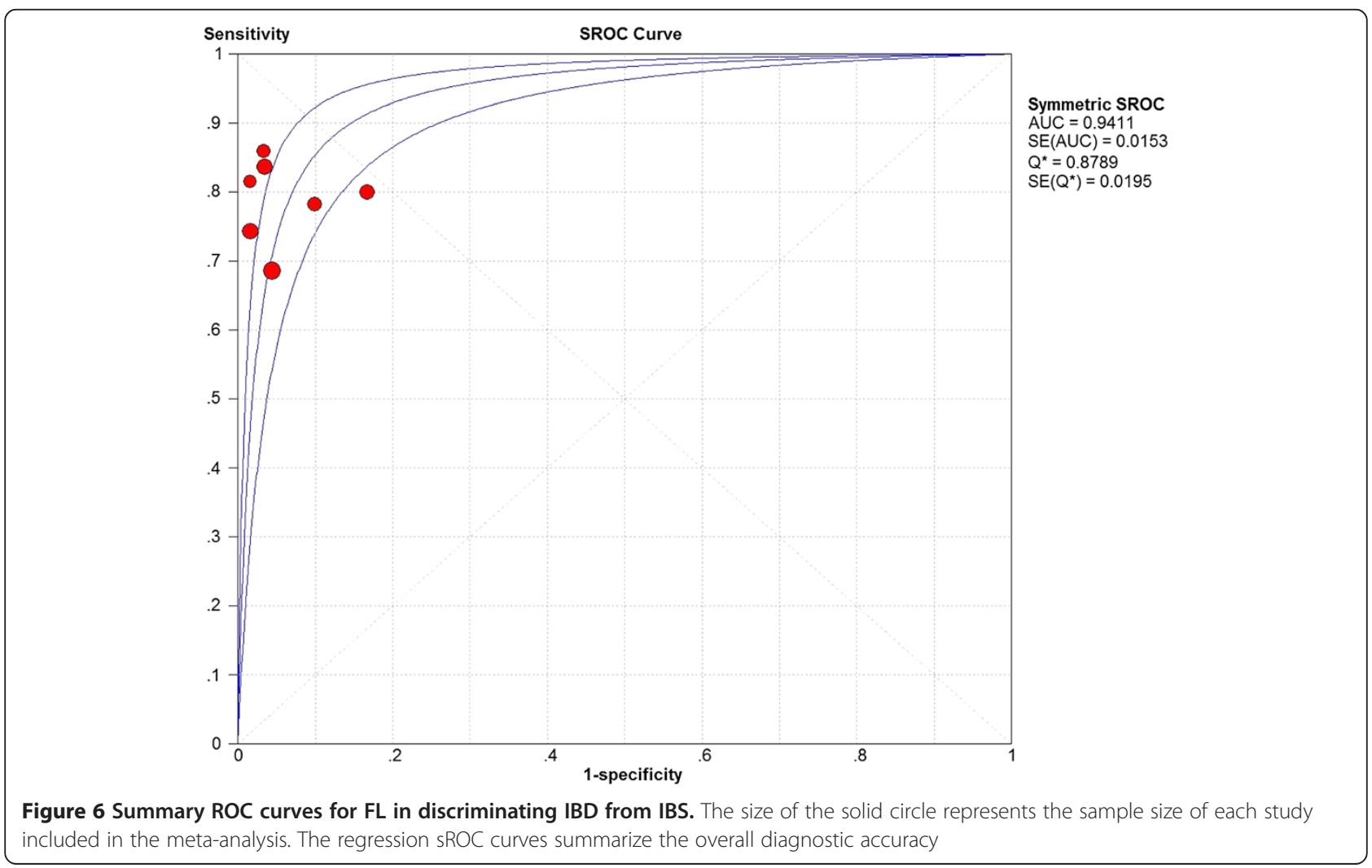


IBS. The pooled sROC showed an AUC of 0.94, indicating a promising discriminative ability. The DOR, defined as the ratio of the odds of positive test results between the diseased and non diseased groups, with higher values indicating greater accuracy, was 52.65 [35]. Likelihood ratio is a metric that incorporates both the sensitivity and specificity, is less affected by prevalence, and is widely considered more useful in clinical practice [36]. Although there is no absolute threshold, a good diagnostic test may have a PLR $>5$ and an NLR $<0.2$ [37]. We observed a PLR of 12.31, indicating that patients with IBD had an approximately 12.31-fold higher chance of testing positive than patients with IBS. We also observed an NLR of 0.23 , indicating that a patient with IBD had a $23 \%$ chance of testing negative.

The 1012 patients analyzed in our study included both children and adults. Although FL has been reported to vary by age in healthy volunteers [38], the results from the seven included studies showed no significant difference between children and adults. These findings suggested that FL assays could be utilized in a wide spectrum of age groups. Most of the included studies [3,10-14] contained patients with IBD of different disease severity, with FL being a more sensitive assay in patients with active than inactive IBD. Because the data were insufficient and the various studies utilized different clinical activity indices, our analysis failed to stratify patients by disease activity. Nevertheless, several studies have found that FL levels are higher in patients with inactive IBD than in patients with IBS [10,12-14].

Ulcerative colitis (UC) and Crohn's disease (CD) are two types of IBD with different inflammatory patterns. UC is primarily characterized by superficial inflammation of the colon with neutrophil infiltration, the obligatory involvement of the rectum in some adult patients or the left colon, together with superficial inflammation, leading to a short transit time of released FL. In contrast, patients with CD show small intestinal involvement, with a longer transit time and/or the accumulation of inflammatory cells in deeper mucosal layers possibly resulting in FL levels being higher in CD than in UC $[4,39]$. In contrast, one study reported higher FL concentrations in CD [13], and another showed that FL levels tended to be higher in patients with isolated colonic disease than in patients with involvement of the ileum alone or the ileocolon [3].Owing to data limitations, we did not perform subgroup analysis based on disease type and location.

Our study had several limitations. First, all studies included in our meta-analysis were from western countries, which may have biased our findings. Second, our meta-analysis was based on published studies; the exclusion of unpublished data (grey literature) is generally associated with an overestimation of the true effect, thus resulting in a publication bias. Third, the reliability of the pooled estimates was dependent on the methodological quality of the included studies. Although eligible studies met many of the QUADAS criteria, weaknesses remained. Fourth, some degree of heterogeneity was introduced by the variability in patient characteristics. These individuals had different types of IBD, different disease activity and different sites of inflammation. Owing to the small number of people included in this meta-analysis and the lack of available information, we did not perform subgroup analysis by pretest probability of CD or UC (based on clinical assessment), or by disease severity or distribution. Fifth, one study included in our meta-analysis pooled data from IBS patients and healthy controls. Since healthy individuals were not representative of the study population, it may have generated some heterogeneity. Sixth, although all included studies measured FL using the same assay, the thresholds varied, perhaps owing to differences in subject profile or inclusion or exclusion criteria. Therefore, we could not determine an optimum cutoff value for FL.

\section{Conclusions}

This meta-analysis showed that FL appears to have good diagnostic precision in distinguishing IBD from IBS both in adults and children. Owing to study limitations, additional high-quality original studies (especially in patients stratified by disease type, severity and distribution) are required to confirm the predictive value of FL.

\section{Competing interests}

The authors declare that they have no competing interests.

\section{Authors' contributions}

WP, XX and QWcontributed to the conception, design and final approval of the submitted version. $X Z, W X$ and $X T$ developed the literature search, carried out the extraction of data, assisted in the critical appraisal of included studies and assisted in writing up. LL, JT and CZ carried out the statistical analysis of studies. All authors read and approved the final manuscript.

\section{Acknowledgments}

This research was supported by grants from the National Health Key Special Fund (No.200802112), the National Science Fund Committee

(No.81372302,81272120), the Health Department Fund (No. 2007A093), the Key Project of Zhejiang Province (No.2013C03044-5,2009C030125), the Natural Science Fund of Zhejiang Province (No. Y2080001,Y12H160121 and Z2080514) and the Traditional Chinese Medicine Bureau Fund (No. 2007ZA019)

\section{Author details}

${ }^{1}$ Department of Gastroenterology, Second Affiliated Hospital of Zhejiang University, School of Medicine, 88 Jiefang Road, Hangzhou, China.

${ }^{2}$ Department of Pharmacy, Second Affiliated Hospital of Zhejiang University, School of Medicine, 88 Jiefang Road, Hangzhou, China.

Received: 13 February 2014 Accepted: 3 July 2014

Published: 7 July 2014

\section{References}

1. Bernstein CN, Fried M, Krabshuis JH, Cohen H, Eliakim R, Fedail S, Gearry R, Goh KL, Hamid S, Khan AG, LeMair AW, Malfertheiner, Ouyang Q, Rey JF, Sood A, Steinwurz F, Thomsen OO, Thomson A, Watermeyer G: World Gastroenterology Organization Practice Guidelines for the 
diagnosis and management of IBD in 2010. Inflamm Bowel Dis 2010, 16(1):112-124.

2. Quigley EM, Abdel-Hamid H, Barbara G, Bhatia SJ, Boeckxstaens G, De Giorgio R, Delvaux M, Drossman DA, Foxx-Orenstein AE, Guarner F, Gwee KA, Harris LA, Hungin AP, Hunt RH, Kellow JE, Khalif IL, Kruis W, Lindberg G, Olano C, Moraes-Filho JP, Schiller LR, Schmulson M, Simren M, Tzeuton C: A global perspective on irritable bowel syndrome: a consensus statement of the World Gastroenterology Organisation Summit Task Force on irritable bowel syndrome. J Clin Gastroenterol 2012, 46(5):356-366.

3. Schroder O, Naumann M, Shastri Y, Povse N, Stein J: Prospective evaluation of faecal neutrophil-derived proteins in identifying intestinal inflammation: combination of parameters does not improve diagnostic accuracy of calprotectin. Aliment Pharmacol Ther 2007, 26(7):1035-1042.

4. Langhorst J, Elsenbruch S, Mueller T, Rueffer A, Spahn G, Michalsen A, Dobos GJ: Comparison of 4 neutrophil-derived proteins in feces as indicators of disease activity in ulcerative colitis. Inflamm Bowel Dis 2005, 11(12):1085-1091.

5. Poullis A, Foster R, Northfield TC, Mendall MA: Review article: faecal markers in the assessment of activity in inflammatory bowel disease. Aliment Pharmacol Ther 2002, 16(4):675-681.

6. Roseth AG, Schmidt PN, Fagerhol MK: Correlation between faecal excretion of indium-111-labelled granulocytes and calprotectin, a granulocyte marker protein, in patients with inflammatory bowel disease. Scand J Gastroenterol 1999, 34(1):50-54.

7. Brock JH: Lactoferrin-50 years on. Biochem Cell Biol 2012, 90(3):245-251.

8. Kopylov U, Rosenfeld G, Bressler B, Seidman E: Clinical utility of fecal biomarkers for the diagnosis and management of inflammatory bowel disease. Inflamm Bowel Dis 2014, 20(4):742-756.

9. Guerrant RL, Araujo V, Soares E, Kotloff K, Lima AA, Cooper WH, Lee AG: Measurement of fecal lactoferrin as a marker of fecal leukocytes. J Clin Microbiol 1992, 30(5):1238-1242.

10. Walker TR, Land ML, Kartashov A, Saslowsky TM, Lyerly DM, Boone JH, Rufo PA: Fecal lactoferrin is a sensitive and specific marker of disease activity in children and young adults with inflammatory bowel disease. J Pediatr Gastroenterol Nutr 2007, 44(4):414-422.

11. Schoepfer AM, Trummler M, Seeholzer P, Seibold-Schmid B, Seibold F: Discriminating IBD from IBS: comparison of the test performance of fecal markers, blood leukocytes, CRP, and IBD antibodies. Inflamm Bowel Dis 2008, 14(1):32-39.

12. Langhorst J, Elsenbruch S, Koelzer J, Rueffer A, Michalsen A, Dobos GJ: Noninvasive markers in the assessment of intestinal inflammation in inflammatory bowel diseases: performance of fecal lactoferrin, calprotectin, and PMN-elastase, CRP, and clinical indices. Am J Gastroenterol 2008, 103(1):162-169.

13. Kane SV, Sandborn WJ, Rufo PA, Zholudev A, Boone J, Lyerly D, Camilleri M Hanauer SB: Fecal lactoferrin is a sensitive and specific marker in identifying intestinal inflammation. Am J Gastroentero/ 2003, 98(6):1309-1314.

14. Sidhu R, Wilson P, Wright A, Yau CW, D'Cruz FA, Foye L, Morley S, Lobo AJ, McAlindon ME, Sanders DS: Faecal lactoferrin-a novel test to differentiate between the irritable and inflamed bowel? Aliment Pharmacol Ther 2010, 31(12):1365-1370.

15. Otten CM, Kok L, Witteman BJ, Baumgarten R, Kampman E, Moons KG, de Wit NJ: Diagnostic performance of rapid tests for detection of fecal calprotectin and lactoferrin and their ability to discriminate inflammatory from irritable bowel syndrome. Clin Chem Lab Med 2008, 46(9):1275-1280.

16. Joishy M, Davies I, Ahmed M, Wassel J, Davies K, Sayers A, Jenkins H: Fecal calprotectin and lactoferrin as noninvasive markers of pediatric inflammatory bowel disease. J Pediatr Gastroenterol Nutr 2009, 48(1):48-54.

17. Silberer H, Kuppers B, Mickisch O, Baniewicz W, Drescher M, Traber L, Kempf A, Schmidt-Gayk H: Fecal leukocyte proteins in inflammatory bowel disease and irritable bowel syndrome. Clin Lab 2005, 51(3-4):117-126.

18. Dai J, Liu WZ, Zhao YP, Hu YB, Ge ZZ: Relationship between fecal lactoferrin and inflammatory bowel disease. Scand J Gastroenterol 2007, 42(12):1440-1444.

19. Whiting PF, Weswood ME, Rutjes AW, Reitsma JB, Bossuyt PN, Kleijnen J: Evaluation of QUADAS, a tool for the quality assessment of diagnostic accuracy studies. BMC Med Res Methodol 2006, 6:9.

20. Leeflang MM, Deeks JJ, Gatsonis C, Bossuyt PM: Systematic reviews of diagnostic test accuracy. Ann Intern Med 2008, 149(12):889-897.
21. Higgins JP, Thompson SG, Deeks JJ, Altman DG: Measuring inconsistency in meta-analyses. BMJ 2003, 327(7414):557-560.

22. DerSimonian R, Laird N: Meta-analysis in clinical trials. Control Clin Trials 1986, 7(3):177-188

23. Egger M, Davey Smith G, Schneider M, Minder C: Bias in meta-analysis detected by a simple, graphical test. BMJ 1997, 315(7109):629-634.

24. Gisbert JP, McNicholl AG, Gomollon F: Questions and answers on the role of fecal lactoferrin as a biological marker in inflammatory bowel disease. Inflamm Bowel Dis 2009, 15(11):1746-1754.

25. Lundberg JO, Hellstrom PM, Fagerhol MK, Weitzberg E, Roseth AG: Technology insight: calprotectin, lactoferrin and nitric oxide as novel markers of inflammatory bowel disease. Nat Clin Pract Gastroenterol Hepatol 2005, 2(2):96-102.

26. Sipponen T, Savilahti E, Kolho KL, Nuutinen H, Turunen U, Farkkila M: Crohn's disease activity assessed by fecal calprotectin and lactoferrin: correlation with Crohn's disease activity index and endoscopic findings. Inflamm Bowel Dis 2008, 14(1):40-46.

27. Jones J, Loftus EV Jr, Panaccione R, Chen LS, Peterson S, McConnell J, Baudhuin L, Hanson K, Feagan BG, Harmsen SW, Zinsmeister AR, Helou E, Sandborn WJ: Relationships between disease activity and serum and fecal biomarkers in patients with Crohn's disease. Clin Gastroenterol Hepatol 2008, 6(11):1218-1224.

28. Xiang JY, Ouyang Q, Li GD: [Significance of fecal lactoferrin in evaluation of disease activity in ulcerative colitis]. Zhonghua Yi Xue Za Zhi 2007, 87(32):2262-2264.

29. D'Inca R, Dal Pont E, Di Leo V, Ferronato A, Fries W, Vettorato MG, Martines D, Sturniolo GC: Calprotectin and lactoferrin in the assessment of intestinal inflammation and organic disease. Int J Colorectal Dis 2007, 22(4):429-437.

30. Buderus S, Boone J, Lyerly D, Lentze MJ: Fecal lactoferrin: a new parameter to monitor infliximab therapy. Dig Dis Sci 2004, 49(6):1036-1039.

31. Sipponen $T$, Savilahti $E$, Karkkainen $P$, Kolho $K L$, Nuutinen $H$, Turunen $U$, Farkkila M: Fecal calprotectin, lactoferrin, and endoscopic disease activity in monitoring anti-TNF-alpha therapy for Crohn's disease. Inflamm Bowel Dis 2008, 14(10):1392-1398

32. Sutherland AD, Gearry RB, Frizelle FA: Review of fecal biomarkers in inflammatory bowel disease. Dis Colon Rectum 2008, 51(8):1283-1291.

33. Schoepfer AM, Trummler M, Seeholzer P, Criblez DH, Seibold F: Accuracy of four fecal assays in the diagnosis of colitis. Dis Colon Rectum 2007, 50(10):1697-1706.

34. Burri E, Manz M, Rothen C, Rossi L, Beglinger C, Lehmann FS: Monoclonal antibody testing for fecal calprotectin is superior to polyclonal testing of fecal calprotectin and lactoferrin to identify organic intestinal disease in patients with abdominal discomfort. Clin Chim Acta 2013, 416:41-47.

35. Glas AS, Lijmer JG, Prins MH, Bonsel GJ, Bossuyt PM: The diagnostic odds ratio: a single indicator of test performance. J Clin Epidemiol 2003, 56(11):1129-1135

36. McGee S: Simplifying likelihood ratios. J Gen Intern Med 2002, 17(8):646-649.

37. Deeks JJ: Systematic reviews in health care: Systematic reviews of evaluations of diagnostic and screening tests. BMJ 2001, 323(7305):157-162.

38. Joshi S, Lewis SJ, Creanor S, Ayling RM: Age-related faecal calprotectin, lactoferrin and tumour M2-PK concentrations in healthy volunteers. Ann Clin Biochem 2010, 47(Pt 3):259-263.

39. Sugi K, Saitoh O, Hirata I, Katsu K: Fecal lactoferrin as a marker for disease activity in inflammatory bowel disease: comparison with other neutrophil-derived proteins. Am J Gastroenterol 1996, 91(5):927-934.

doi:10.1186/1471-230X-14-121

Cite this article as: Zhou et al:: Fecal lactoferrin in discriminating

inflammatory bowel disease from Irritable bowel syndrome: a diagnostic meta-analysis. BMC Gastroenterology 2014 14:121. 